\title{
The impact of diabetes mellitus at the time of heart transplantation on long-term survival
}

\author{
M. Czerny ${ }^{1}$, V. Sahin 1 , P. Fasching ${ }^{2}$, A. Zuckermann'1, D. Zimpfer ${ }^{1}$, J. Kilo ${ }^{1}$, E. Wolner ${ }^{1}$, M. Grimm ${ }^{1}$ \\ ${ }^{1}$ Department of Cardio-thoracic Surgery, University of Vienna Medical School, Vienna, Austria \\ $23^{\text {rd }}$ Department of Internal Medicine, Geriatric Centre Baumgarten, Vienna, Austria
}

\section{Abstract}

Aims/hypothesis. To analyse the impact of diabetes mellitus (DM) at the time of heart transplantation on long-term survival and incidence of transplant coronary artery disease (TxCAD).

Methods. We analysed 773 consecutive adult heart transplant recipients who underwent primary heart transplantation from May 1986 until December 2000. The cohort consisted of 140 patients with diabetes mellitus (with DM, men 82\%) and 633 patients without (wo DM, men 84\%) diabetes mellitus at the time of transplantation. The patients were documented as to survival and incidence of TxCAD.

Results. Patients with diabetes mellitus were older compared to those without diabetes mellitus (with DM 54.9 $\pm 6.8 \mathrm{a}$ vs wo DM 49.7 $\pm 10.8 \mathrm{a} ; p=0.0001$ ), they had a higher incidence of ischaemic cardiomyopathy prior to transplantation (with DM $52 \%$ vs wo DM 30\%; $p=0.0001$ ), but reduced long-term survival (10 year survival: with DM 40\% vs wo DM 58\%; logrank=0.025). Surprisingly, the incidence of transplant coronary artery disease (TxCAD) was comparable at 10 years (with DM $28 \%$ vs wo DM 22\%; logrank=0.625). In multivariate Cox proportional hazard analysis, diabetes mellitus present at the time of heart transplantation (HR 1.594; 95\%CI 1.009-2.518; $p=0.045$ ), but not age (HR 0.990; 95\%CI 0.965$1.014 ; p=0.404)$ was an independent predictor affecting long-term survival.

Conclusion/interpretation. The presence of diabetes mellitus at the time of heart transplantation adversely affects long-term patient survival, but does not predict the occurrence of transplant coronary artery disease. The definite mechanisms of adverse survival primarily seem to relate to generally impaired global organ function. Despite a less favourable long-term outcome, our data still justify heart transplantation in end-stage heart failure patients with diabetes mellitus. [Diabetologia (2002) 45:1498-1508]

Keywords: Diabetes mellitus, heart transplantation, survival, transplant coronary artery disease
Received: 6 May 2002 / Revised: 15 July 2002

Published online: 18 October 2002

(C) Springer-Verlag 2002

Corresponding author: M. Grimm, MD, Division of Cardiothoracic Surgery, Department of Surgery, Waehringer Guertel 18-20, A-1090 Vienna, Austria.

E-mail: michael.grimm@akh-wien.ac.at

Abbreviations: DM, Diabetes mellitus; TxCAD, transplant coronary artery disease; CMV, cytomegalovirus; ISHLT, International Society for Heart and Lung Transplantation; IVUS, intravascular ultrasound; LAD, left anterior descending artery.
The presence of diabetes mellitus has long been considered a contraindication to heart transplantation because corticosteroid immunosuppressive therapy exacerbates hyperglycaemia and increases the potential for infection particularly in patients with diabetes $[1,2,3$, 4]. The introduction of cyclosporine-based immunosuppressive therapy permitted the reduction of individual steroid dosage needed and thereby reduced the likelihood of a steroid-based exacerbation of diabetes [5]. Subsequently, the initial reluctance to accept patients with diabetes as candidates for heart transplantation has reduced and encouraging results have been reported. No major differences in the patients' short- 
term survival, rate of infection, rate of transplant rejection, renal function as well as transplant coronary artery disease as compared with patients without diabetes were reported in limited series $[4,5]$. Due to improved as well as extended conventional therapy of patients with diabetes (especially with ischaemic heart disease) the number of patients with diabetes and endstage ischaemic cardiomyopathy is likely to increase in the near future $[6,7,8]$. Therefore, a risk stratification for patients with diabetes eligible for heart transplantation is strongly needed to clarify long-term prognosis.

The long-term outcome in patients with diabetes at the time of heart transplantation is still uncertain. It was the aim of this retrospective single-centre study to analyse the impact of diabetes mellitus at the time of heart transplantation on long-term survival and incidence of transplant coronary artery disease.

\section{Subjects and methods}

Patients. A retrospective analysis was done in 773 consecutive adult heart transplant recipients who underwent their primary transplantation from May 1986 until December 2000 (Table 1). The cohort consisted of 140 patients with diabetes mellitus (DM) (with DM, men $n=115,82 \%$ ) and 633 patients without (wo DM, men $n=532,84 \%$ ) diabetes at the time of transplantation. Of the 140 patients with diabetes (men $n=9,7 \%$ ) ten had Type I (insulin-dependent) diabetes mellitus with the defined onset of the disease at an age of 35 years or younger with an immediate need for insulin substitution. Median observation time was $69 \pm 3$ months in patients with diabetes and $84 \pm 2$ months in patients with out diabetes at the time of transplantation $(p=0.143)$.

Patients with diabetes were evaluated for heart transplantation on an individual basis. A detailed work-up was carried out in patients with a known duration of diabetes for more than 10 years to analyse the extent of diabetes-related complications. It included an eye examination, creatinine clearance test as well as a complete peripheral arterial status including carotid artery ultrasound sonography and lower limb ultrasound sonography.

Immunosuppressive protocol: cytolytic induction therapy. All patients received cytolytic induction therapy with either polyclonal antibodies (thymoglobuline $n=513$, IMTIX-SangStat, Lyon, France, $2.5 \mathrm{mg} / \mathrm{kg}$ /day for 7 days; ATG-fresenius $n=177$, Fresenius, Bad Homburg, Germany, $10 \mathrm{mg} / \mathrm{kg} /$ day for 7 days; ATGAM $n=21$, Upjohn Pharmaceuticals; Tokyo, Japan, $10 \mathrm{mg} / \mathrm{kg} /$ day for 7 days) or monoclonal antibodies (BT563 $n=27$, Biotest, Dreireich, Germany, $3 \mathrm{mg} / \mathrm{kg} /$ day for 7 days; OKT3 $n=35$, Roche Laboratories, New Jersey, USA, 5 mg/day for 7 days).

Maintainance therapy: calcineurin antagonists. Calcineurin antagonist therapy (Cyclosporine A - based $n=737$, Tacrolimus - based $n=36$ ) was begun intravenously if the transplant recipient was haemodynamically stable after the operation and was progressively adjusted to a standardised target concentration (Cyclosporine A - 200-400 ng/ml and Tacrolimus 10-15 ng/ml, respectively). Cyclosporine A and Tacrolimus dosage adjustments were guided by the maximum acceptable creatinine concentrations of 150 to $170 \mathrm{mmol} / \mathrm{l}$. Between the
Table 1. Patients with and without diabetes mellitus at the time of transplantation - diagnoses and perioperative data

\begin{tabular}{lccl}
\hline $\mathrm{c}$ & With DM & Without DM & $p$ \\
\hline$n$ & 140 & 633 & \\
Age at transplantation & $54.9 \pm 6.8$ & $49.7 \pm 10.8$ & 0.0001 \\
$\quad$ (years) & & & \\
Donor age (years) & $31.4 \pm 7.9$ & $31.8 \pm 9.3$ & 0.609 \\
Male recipients (\%) & 82 & 84 & 0.426 \\
Male donors (\%) & 71 & 68 & 0.354 \\
Serum creatinine preTX & $107 \pm 51$ & $108 \pm 61$ & 0.509 \\
$\quad$ (mmol/1) & & & \\
Diagnosis (\%) & & & \\
$\quad$ coronary artery disease & 52 & 30 & 0.0001 \\
$\quad$ idiopathic & 46 & 65 & 0.101 \\
$\quad$ others & 2 & 5 & 0.303 \\
Ischaemic time (min) & $171 \pm 57$ & $160 \pm 58$ & 0.779 \\
Reoperation (\%) & 26 & 23 & 0.521 \\
Perioperative mortality (\%) & 13 & 11 & 0.306 \\
Cyclosporine A-based & 18 & 82 & \\
$\quad$ immunosuppression (\%) & & & \\
Tacrolimus-based & 12 & 88 & \\
$\quad$ immunosuppression (\%) & & & \\
\hline
\end{tabular}

$2^{\text {nd }}$ and $6^{\text {th }}$ month after transplantation Cyclosporine A target concentrations were 150 to $250 \mathrm{ng} / \mathrm{ml}$ and 100 to $150 \mathrm{ng} / \mathrm{ml}$ thereafter. Tacrolimus target concentrations were 8 to $12 \mathrm{ng} / \mathrm{ml}$ and 5 to $10 \mathrm{ng} / \mathrm{ml}$ thereafter, respectively. The azathioprine dose ( $2 \mathrm{mg} / \mathrm{kg} /$ day) was adjusted to a leukocyte count between 4000 to 6000 cells per $\mathrm{mm}$.

Steroids were given i.v. (methylprednisolone $500 \mathrm{mg}$ before reperfusion of the allograft) and continued every $8 \mathrm{~h}$ for an additional three $125 \mathrm{mg}$ doses. Oral prednisolone $(1 \mathrm{mg} / \mathrm{kg})$ was started on day 2 and tapered to a maintenance dose of 0.2 to $0.15 \mathrm{mg}$ per $\mathrm{kg}$. In the case of an immunologically uneventful 6 month postoperative course, steroids were further reduced to $0.1 \mathrm{mg}$ per $\mathrm{kg}$.

Infectious prophylaxis. Antistaphylococcal antibiotics were given for 5 days plus $100 \mathrm{ml}$ anti-cytomegalovirus (CMV) hyperimmunoglobuline (Cytotect, Biotest, Dreireich, Germany) immediately before transplantation and on days 1, 7, 14, 21 and 28 thereafter. In addition $100 \mathrm{ml}$ oral nystatin was given daily for 28 days.

Surveillance of infection. Screening for bacterial infection was by routine bacterial cultures from blood, urine and tracheobronchial secretions on days 1, 3, 5, 7, 14, 21 and 28. Viral infection was surveyed by serological analysis (IgG and $\mathrm{IgM}$ ) for CMV, herpes simplex, varicella zoster, and Epstein-Barr virus and by documentation of CMV immediate early antigen in fibroblast culture incubated with leucocytes, urine, and oropharyngeal washings on days $7,14,21,28$ and monthly from months 2 to 6 . Further studies were carried out whenever clinical signs of infection occurred. CMV disease was diagnosed if CMV infection was associated with (i) fever $\left(>38^{\circ} \mathrm{C}\right)$, (ii) influenza-like symptoms with or without pneumonitis, colitis, hepatitis, or carditis, (iii) leukopaenia (leukocyte count $<3.5 \times 10^{9}$ cells $/ 1$ on two consecutive measurements after stopping azathioprine therapy), and (iv) absence of any other infection or rejection.

Assessment of rejection. The International Society of Heart and Lung Transplantation (ISHLT) grading system was used to de- 
tect cardiac rejection in serial endomyocardial biopsies [9]. Rejection was defined as either a grade equal to 2 or a grade less than 2 with haemodynamic compromise. Biopsies were carried out weekly during the first month, monthly in the first 6 months, and once at the end of the first year after transplantation or as clinically indicated.

Antirejection therapy. Rejection episodes were treated with methylprednisolone (15 mg/kg i.v. daily for 3 days) followed by a control biopsy after 1 week. If steroid therapy failed, a 10day course of $5 \mathrm{mg}$ OKT-3 was initiated.

Surveillance of transplant coronary artery disease (TxCAD). We have used the standardized international angiographic categorization of allograft vascular disease [10]. This categorization defines TxCAD class as mild (left main $<50 \%$, a maximum lesion of $<70 \%$ in primary vessels or an isolated single-branch stenosis $>70 \%$ ), moderate (left main $50-70 \%$, two or more primary vessels $>70 \%$ or an isolated branch stenosis $>70 \%$ in branches of two systems) or severe (left main $>70 \%$, two or more primary vessels $>70 \%$ or isolated branch stenosis $>70 \%$ in all 3 systems). All patients underwent quantitative coronary angiography as well as intravascular ultrasounds (IVUS) at 12, 36 and 60 months after heart transplantation and annually thereafter. Standard IVUS measurements were obtained with computerized planimeters [11]. Angiograms and IVUS were also carried out, whenever the clinical situation suggested occurrence of TxCAD.

Glucose control. Adequate glucose control was achieved in patients with diabetes by dietary management for which they received intensive instruction, and by pharmacologic management. Dietary means, oral hypoglycaemic drug doses (sulphonylureas, metformin) or insulin were adjusted to maintain fasting blood glucose concentrations between $6.7 \mathrm{mmol} / \mathrm{l}$ and at least $11.1 \mathrm{mmol} / 1$.

Statistical analysis. All the clinical or laboratory data are expressed as mean and standard deviation. Data processing and statistical analysis were carried out using SAS statistical software. The nonparametric Mann-Whitney $U$ test was used to calculate differences between the two groups. The chi-square test was used to compare categorical variables. Groups were compared by actuarial Kaplan-Meier event free survival calculations and statistical testing using the Wilcoxon rank sum test and log-rank test for the following clinical endpoints: death, acute clinical rejection (grade 2 ISHLT or $<2$ ISHLT with haemodynamic compromise), infectious episodes as well as TxCAD. Univariate analysis and Cox proportional hazards models were carried out for patient survival and TxCAD. Patient, donor and characteristics of cytolytic induction therapy were studied for their impact on survival and development of TxCAD. A $p$ value less than 0.05 was considered significant.

\section{Results}

Patient characteristics. Patient characteristics at the time of heart transplantation are shown in Table 1. Patients with diabetes were older compared with patients without diabetes (with DM 54.9 $\pm 6.8 \mathrm{a}$ vs wo DM $49.7 \pm 10.8 \mathrm{a} ; p=0.0001)$ and had a higher prevalence of coronary artery disease as indication for transplantation than patients without diabetes (with DM 52\% vs wo DM 30\%; $p=0.0001$ ).
Survival. Long-term survival rates of patients who had a transplant and had diabetes were lower compared with patients who had a transplant and did not have diabetes (5 year survival: with DM $57.1 \%$ vs wo DM 67.6\%; 10 year survival: with DM $40 \%$ vs wo DM 58\%; log-rank=0.025; Fig. 1A). Causes of death were proportional in both groups over the whole study period: Infections (with DM $31.0 \%$ vs wo DM 28.4\%; $\mathrm{p}=0.583$ ); TxCAD (with DM $12.1 \%$ vs wo DM 12.4\%; $p=0.167$ ); malignancies (with DM 17.2\% vs wo DM $13.8 \% ; p=0.406$ ) as well as episodes of acute rejection (with DM 6.9\% vs wo DM 12.9\%; $p=0.588$ ) were comparable.

In patients who had a transplant and had diabetes, those who were treated by oral hypoglycaemic agents (sulphonylureas, metformin) at the time of transplantation, showed a clear trend towards adverse survival compared with patients initially treated by dietary means or insulin (log-rank=0.068) (Fig. 2A).

Transplant coronary artery disease. Both patient groups showed the same incidence of TxCAD documented by quantitative coronary angiography as well as by intravascular ultrasound (TxCAD at 5 years: with DM $15 \%$ vs wo DM 14\%; TxCAD at 10 years: with DM $28 \%$ vs wo DM 22\%; log-rank=0.167; Fig. 1B). We did not observe any difference in the incidence of TxCAD with regard to the treatment modality of diabetes at the time of transplantation (Fig. 2B). Figure 3A shows a completion angiogram of a patient with diabetes with regular coronary arteries 5 years after transplantation. Figure $3 \mathrm{~B}$ shows a regular mid-left anterior descending artery (LAD) intravascular ultrasound (IVUS) in the same patient. Figure $3 \mathrm{C}$ shows a completion angiogram in a diabetic patient with severe TxCAD 5 years after transplantation with multiple wall irregularities, haemodynamically significant stenoses within the LAD and the circumflex system as well as rarefication of the capillary bed indicating increased peripheral vascular resistance due to microvessel disease. Figure 3D shows midLAD TxCAD as reflected by intimal thickening and a large calcified atherosclerotic plaque in the same patient.

Impact of cytolytic induction therapy. An important impact on long-term survival was observed by cytolytic induction therapy $(\log$-rank $<0.0001)$. Within the group of patients receiving the most potent cytolytic induction therapy (thymoglobuline), we observed superior survival in patients without diabetes compared with patients with diabetes (log-rank=0.012; Fig. 4A). Whereas, within the group of other (less potent) cytolytic induction therapies (polyclonal antibodies - ATG Fresenius, ATGAM; monoclonal antibodies - BT563, OKT 3) overall survival was worse independent of diabetes in this particular group (log-rank=0.504; Fig. 4A). 


\section{A - Survival}

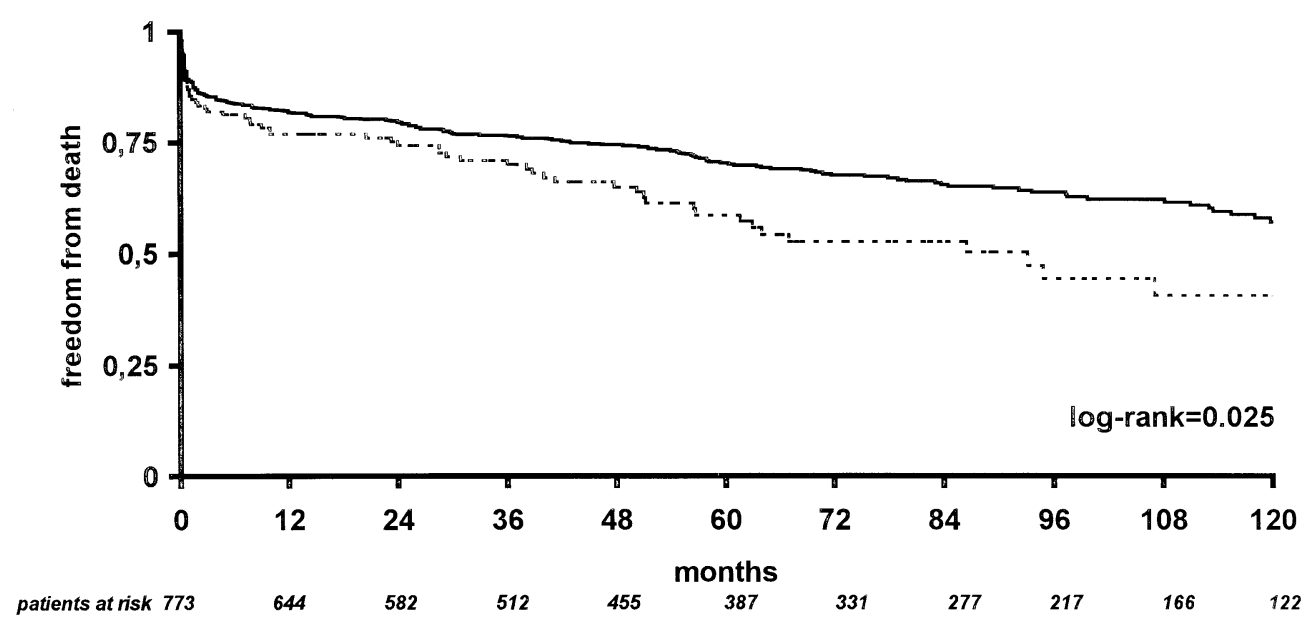

B - TxCAD

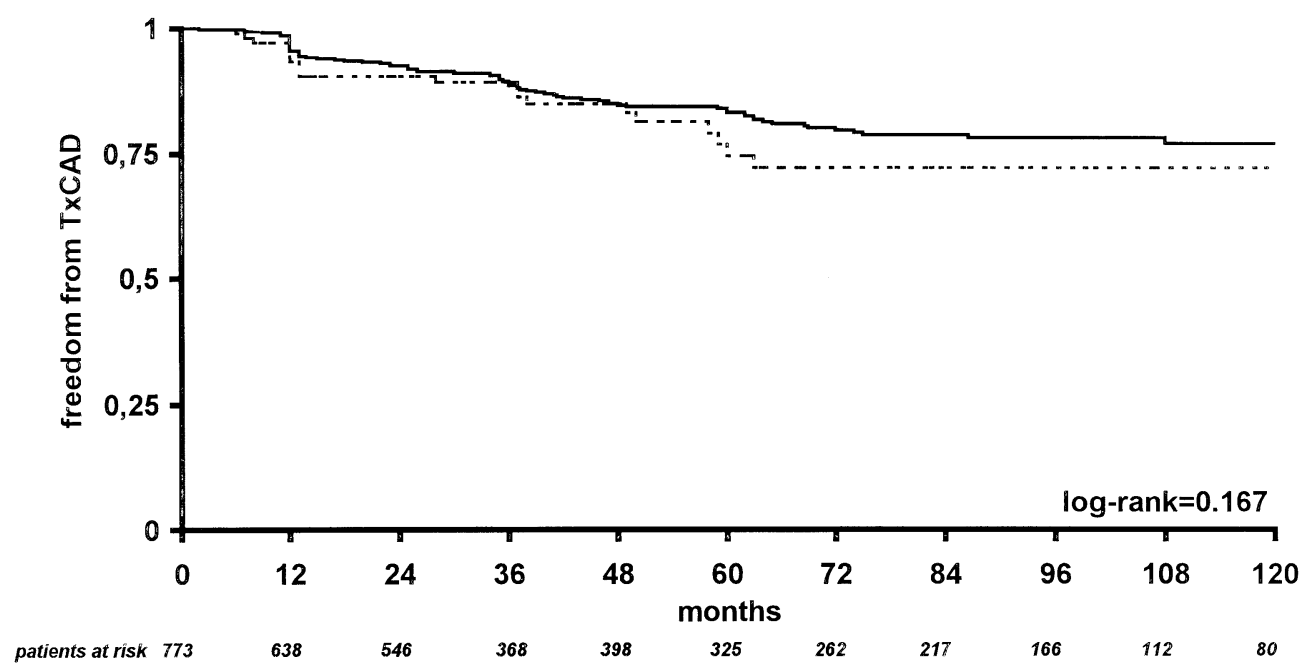

Fig. 1 A, B. Survival (A) and Freedom from TxCAD (B) in patients with (dotted line) and without (solid line) diabetes

In contrast, the use of cytolytic induction therapy with thymoglobuline added an equally beneficial effect in patients with and without diabetes with regard to the incidence of TxCAD (log-rank=0.306; Fig. 4B). Other forms of cytolytic induction therapy (polyclonal antibodies - ATG Fresenius, ATGAM; monoclonal antibodies - BT563, OKT 3) had a higher incidence of TxCAD during follow-up. Nevertheless, within this group of less potent cytolytic induction therapies, patients with diabetes had a higher incidence of TxCAD compared with patients without diabetes (logrank=0.042; Fig. 4B).
Immunosuppressive regimen. Only a minority of patients were treated with Tacrolimus $(n=36)$. Only three of these patients had diabetes at the time of transplantation, we have been using Tacrolimus since 1997. We therefore limited the analysis to find the influence of immunosuppression to 4 years. Mid-term survival rates of patients treated with Cyclosporine A or Tacrolimus were comparable (4-year survival Cyclosporine A based treatment: with DM $75.4 \%$ vs wo DM $80.3 \%$; 4-year survival Tacrolimus - based treatment: with DM $100 \%$ vs wo DM 93.9\%; log-rank=0.192). Additionally, we did not observe any difference with regard to freedom from TxCAD after 4 years (4-year freedom from TxCAD Cyclosporine A - based treatment: with DM $89.7 \%$ vs wo DM 88.4\%; 4-year freedom from TxCAD Tacrolimus - based treatment: with DM $100 \%$ vs wo DM 97.0\%; log-rank=0.617). 


\section{A - Survival with regard to diabetes therapy at the time of $\mathrm{HTX}$}

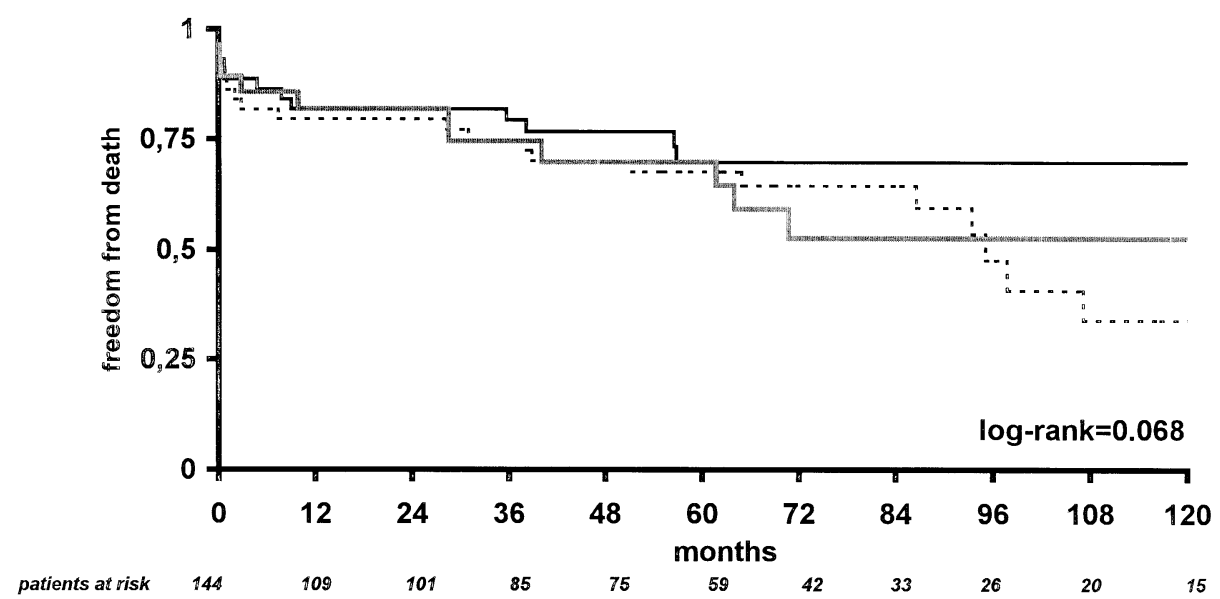

B - TxCAD with regard to diabetes therapy at the time of HTX

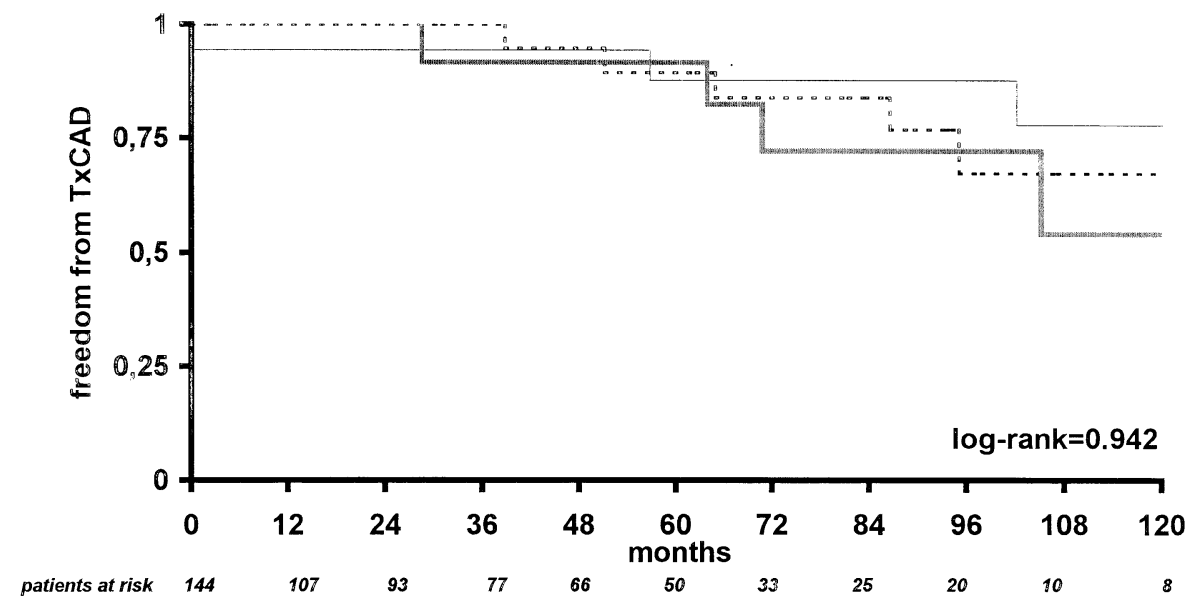

Fig. 2 A, B. Survival (A) and Freedom from TxCAD (B) with regard to diabetes therapy at the time of heart transplantation. Dietary treatment (solid line), oral hypoglycaemic treatment (dotted line), insulin treatment (thick solid line)

Infections. One year after transplantation $60 \%$ of patients with diabetes and $68 \%$ of patients without diabetes had infections in need of therapeutic intervention $(p=0.309)$. After 5 years, infections were observed in $83.2 \%$ of patients with diabetes and in $83.1 \%$ of patients without diabetes $(p=0.415)$. After 10 years, $88 \%$ of patients with diabetes and $92 \%$ of patients without diabetes had infections needing therapeutic intervention $(p=0.644)$. There were no differences with regard to bacterial or viral infections be- tween the two groups. The incidence of CMV infections did not show any difference.

Episodes of acute rejection. Episodes of acute rejection were comparable, $25 \%$ of patients with diabetes and $22 \%$ of patients without diabetes had acute rejections within 5 years $(p=0.432)$. After 10 years, $34 \%$ of patients with diabetes and $33 \%$ of patients without diabetes had rejections $(p=0.791)$. There were no differences with regard to the severity of rejections: Rejections ISHLT $\geq 1 \mathrm{~b}$ were $60 \%$ in patients with diabetes and $62 \%$ in patients without diabetes after 10 years $(p=0.884)$. Rejections ISHLT $\geq 2$ occurred in $26 \%$ of patients with diabetes and $30 \%$ of patients without diabetes after 10 years $(p=0.763)$. 


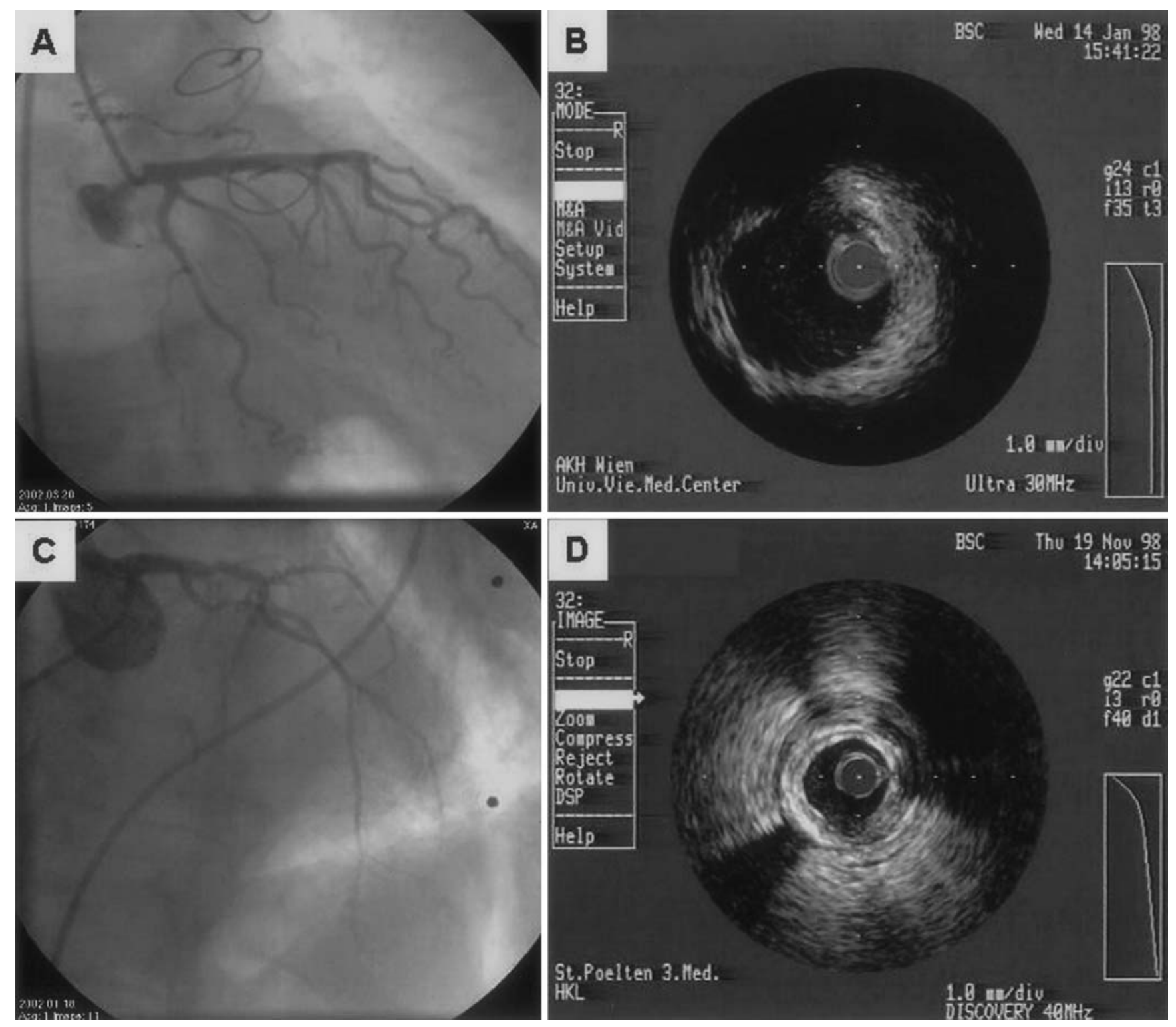

Fig. 3 A. Angiogram with regular coronary arteries after 5 years, B regular IVUS within mid-left LAD after 5 years, $\mathbf{C}$ angiogram with severe TxCAD after 5 years, D mid-LAD TxCAD with intimal thickening and a large calcified atherosclerotic plaque after 5 years

Malignancies. Both groups did not differ in the incidence (with DM $18.1 \%$ vs wo DM $18.0 \%$; $p=0.660$ ) and in the spectrum of tumours they developed. Different kinds of skin cancer (basalioma, Bowen's disease) were the most common sort of cancers (with DM $6.5 \%$ vs wo DM 9.2\%; $p=0.689$ ), followed by lymphoma (with DM $2.6 \%$ vs wo DM $2.6 \% ; p=0.971$ ) and lung cancer (with DM $3.9 \%$ vs wo DM 2.1\%; $p=0.575)$.

Requirement for insulin, $\mathrm{HbA}_{1 \mathrm{c}}$ serum concentrations and serum creatinine concentrations. During follow- up, $15.6 \%$ of all patients who were treated by diet or by oral hypoglycaemic agents at the time of transplantation $(n=106)$ required insulin (Table 2 A). The insulin requirement of those patients already on insulin at the time of heart transplantation $(n=34)$ did not increase within the study period being $49 \pm 18 \mathrm{U}$ before transplantation, $42 \pm 22 \mathrm{IE}$ at 1 year, $44 \pm 24 \mathrm{U}$ at 5 years and $47 \pm 28 \mathrm{U}$ at 10 years after transplantation $(p=0.585)$. Interestingly, mean $\mathrm{HbA}_{1 \mathrm{c}}$ serum concentrations declined after transplantation and did not show an increase during the follow-up period (Table 2 B).

Mean serum creatinine concentrations at the time

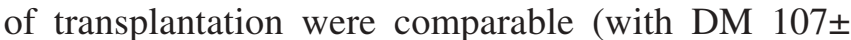
$51 \mathrm{mmol} / \mathrm{l}$ vs wo DM $108 \pm 61 \mathrm{mmol} / \mathrm{l} ; p=0.509)$. The decrease in renal function was also comparable in patients with and without diabetes after 5 years as reflected by a comparable elevation of mean serum creatinine concentrations (with DM $152 \pm 58 \mathrm{mmol} / \mathrm{l}$ vs wo DM $122 \pm 82 \mathrm{mmol} / \mathrm{l} ; p=0.387)$. 


\section{A - Survival with regard to diabetes and cytolytic induction therapy}

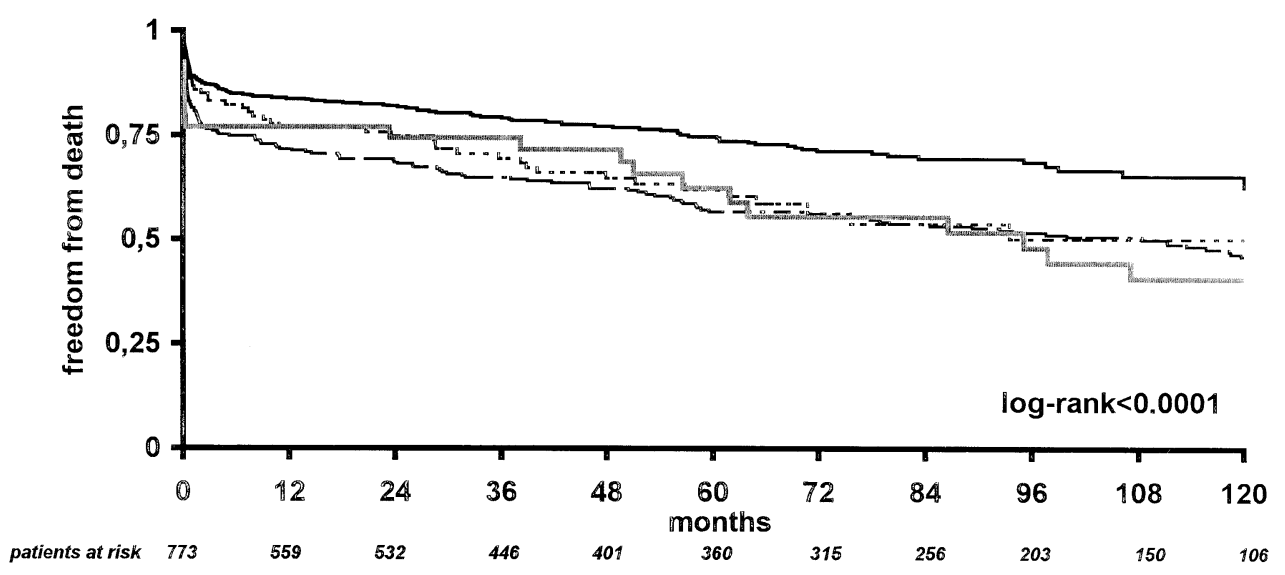

\section{B - TxCAD with regard to diabetes and cytolytic induction therapy}

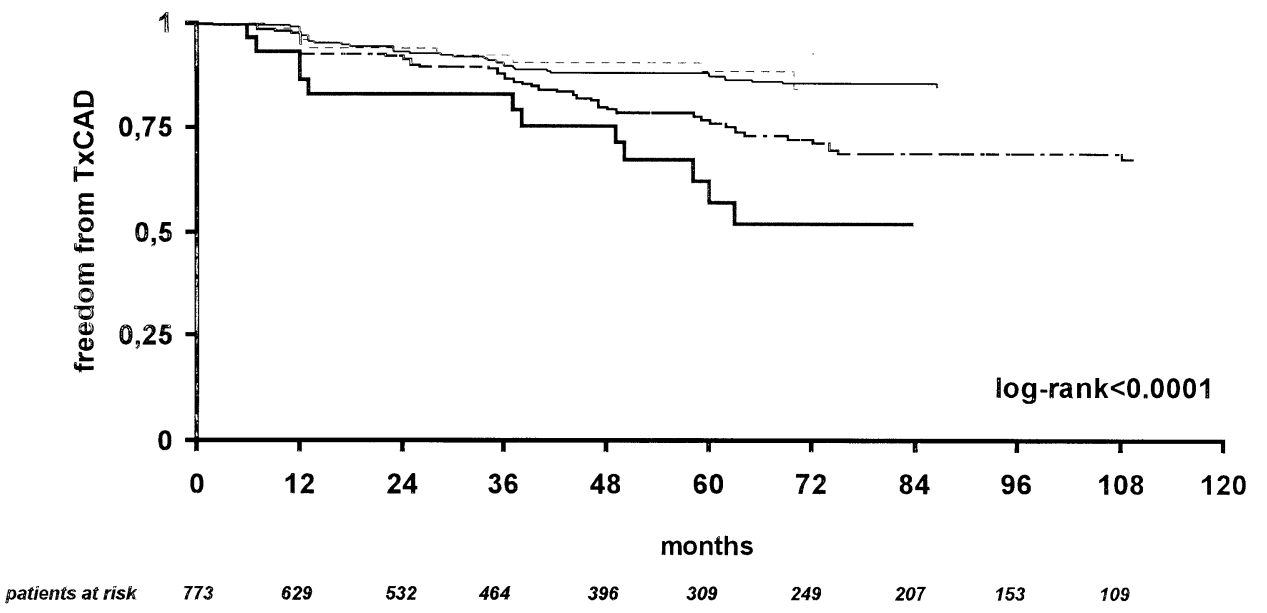

Fig. 4. Survival (A) and Freedom from TxCAD (B) with regard to diabetes and cytolytic induction therapy. Patients with $\mathrm{DM}$ and with cytolytic induction therapy with thymoglobuline (dotted line), patients wo DM and with cytolytic induction therapy with thymoglobuline (solid line), patients with DM and wo cytolytic induction therapy with thymoglobuline (thick solid line), patients wo DM and wo cytolytic induction therapy with thymoglobuline (broken line)

However, after 10 years mean serum creatinine concentrations continued to increase in both groups, but were higher in patients with diabetes compared with patients without diabetes (with DM 164 \pm $90 \mathrm{mmol} / \mathrm{l}$ vs wo DM $143 \pm 72 \mathrm{mmol} / \mathrm{l} ; p=0.032)$.

Univariate predictors of survival and TxCAD. Univariate regression analysis showed diabetes at the time of transplantation $(p=0.032)$, coronary artery disease as indication for transplantation $(p=0.050)$, preoperatively increased serum creatinine concentrations greater than or equal to $160 \mathrm{mmol} / \mathrm{l}(p=0.001)$ as well as cytolytic induction therapy with thymoglobuline $(p<0.0001)$ to be univariate predictors of long-term survival.

Univariate predictors for the development of TxCAD were sex $(p=0.0004)$, coronary artery disease as an indication for transplantation $(p=0.032)$, nicotine abuse ( $p=0.022$ ) as well as cytolytic induction therapy with thymoglobuline $(p=0.039)$. Diabetes did not predict development of TxCAD $(p=0.329)$.

Cox proportional hazards model for predictors of survival and TxCAD. The Cox proportional hazards model showed diabetes at the time of transplantation (HR 1.594; 95\%CI 1.009-2.518; $p=0.045$ ), preoperatively increased serum creatinine concentrations greater than or equal to $160 \mathrm{mmol} / \mathrm{l}$ (HR 1.247; 95\%CI 1.045$1.487 ; p=0.014$ ) as well as the use of cytolytic induc- 
Table 2. Diabetes therapy (A) and HbA1c (B) within study period

\begin{tabular}{lllll}
\hline A - Diabetes therapy & & & \\
\hline Diabetes therapy & Before TX & 1 year after TX & 5 years after TX & 10 years after TX \\
\hline Dietary (\%) & 36.3 & 25.3 & 24.6 & 23.0 \\
Oral (\%) & 39.2 & 35.8 & 36.4 & 36.9 \\
Insulin (\%) & 24.5 & 38.9 & 39.0 & 40.1 \\
\hline
\end{tabular}

$\mathrm{B}-\mathrm{HbA} 1 \mathrm{c}$

\begin{tabular}{llllc}
\hline & Before TX & 1 year after TX & 5 years after TX & 10 years after TX \\
\hline HbA1c $(\%)$ & $8.4 \pm 2.0$ & $7.4 \pm 1.7$ & $7.9 \pm 1.6$ & $8.0 \pm 1.8$ \\
\hline
\end{tabular}

Table 3. Cox proportional hazards model on factors influencing long-term survival

\begin{tabular}{llll}
\hline Variable & Hazard ratio & $95 \%$ Confidence intervals & $p$ \\
\hline Diabetes & 1.594 & $1.009-2.518$ & 0.045 \\
Thymoglobuline & 0.476 & $0.319-0.710$ & 0.0003 \\
Preop serum creatinine $\geq 160 \mathrm{mmol} / \mathrm{l}$ & 1.247 & $1.045-1.487$ & 0.014 \\
Age & 0.996 & $0.978-1.015$ & 0.699 \\
Sex & 0.946 & $0.561-1.596$ & 0.836 \\
Coronary artery disease & 1.333 & $0.897-1.980$ & 0.155 \\
Idiopathic cardiomyopathy & 1.024 & $0.439-2.390$ & 0.955 \\
Ischaemic time & 0.999 & $0.996-1.002$ & 0.435 \\
Nicotine abuse & 0.914 & $0.629-1.329$ & 0.638 \\
BMI & 1.003 & $0.996-1.010$ & 0.452 \\
Insulin dependency & 0.477 & $0.142-1.604$ & 0.231 \\
HbA1c $\geq 8 \%$ & 0.839 & $0.427-1.831$ & 0.319 \\
\hline
\end{tabular}

Table 4. Cox proportional hazards model on factors influencing TxCAD

\begin{tabular}{llll}
\hline Variable & Hazard ratio & $95 \%$ Confidence intervals & $p$ \\
\hline Diabetes & 1.420 & $0.754-2.675$ & 0.277 \\
Thymoglobuline & 0.586 & $0.359-0.957$ & 0.033 \\
Sex & 0.075 & $0.010-0.538$ & 0.010 \\
Age & 0.984 & $0.961-1.007$ & 0.174 \\
Coronary artery disease & 1.413 & $0.874-2.286$ & 0.158 \\
Idiopathic cardiomyopathy & 0.538 & $0.127-2.282$ & 0.400 \\
Ischaemic time & 0.998 & $0.994-1.002$ & 0.405 \\
Preop serum creatinine $\geq 160 \mathrm{mmol} / 1$ & 1.107 & $0.872-1.406$ & 0.402 \\
Nicotine abuse & 1.277 & $0.800-2.037$ & 0.305 \\
BMI & 0.997 & $0.971-1.024$ & 0.839 \\
Insulin dependency & 1.003 & $0.274-3.678$ & 0.995 \\
HbA1c $\geq 8 \%$ & 1.102 & $0.712-2.234$ & 0.751 \\
\hline
\end{tabular}

tion therapy with thymoglobuline (HR $0.476 ; 95 \% \mathrm{CI}$ $0.319-0.710 ; p=0.0003$ ) as independent predictors affecting long-term survival (Table 3). Interestingly, age did not affect long-term survival (HR 0.990; 95\% CI $0.965-1.014 ; p=0.404$ ).

In the Cox proportional hazard model, diabetes did not show any influence on the occurrence of TxCAD
(HR 1.390; 95\%CI 0.739-2.617; $p=0.307$ ). Female sex (HR 0.075; 95\%CI 0.010-0.538; $p=0.012$ ) as well as cytolytic induction therapy with thymoglobuline (HR 0.586; 95\%CI $0.359-0.957 ; p=0.033$ ) were independent predictors affecting the development of TxCAD (Table 4). 


\section{Discussion}

This retrospective, contemporary single centre study shows, that patients, who received a heart transplantation and also had diabetes have a worse long-term survival compared with patients without diabetes. This is very much in contrast to previous reports, not showing any difference in survival $[4,5,11]$. Although this is not surprising, this finding seems to result from a longer follow-up period as well as a larger cohort of patients with diabetes at the time of heart transplantation.

Patients with diabetes were older compared to patients without diabetes and had a higher prevalence of coronary artery disease as an indication to transplantation. Diabetes and preoperatively increased serum creatinine concentrations were, however, neither age nor ischaemic CMP independent predictors of survival. Causes of death were proportional in both, patients with and without diabetes. This is in line with previous reports not showing any difference with regard to causes of death $[4,5]$. As patients with diabetes after renal transplantation present a comparable age and comorbidity matched cohort, we correlated our results to these investigations $[5,12]$. In a study investigating the effect of diabetes on patients after renal transplantation, there was a 12 year survival rate of $48 \%$ in patients with diabetes compared to a rate of $70 \%$ in patients without diabetes [13]. Kaplan-Meier survival curves seemed to show a separation between the groups beginning at about 6 years after renal transplantation. We observed similar trends in our patients with diabetes after heart transplantation, a difference in survival took a while to become evident, first becoming noticeable at 5 years. It seems likely, that early changes are mimicked by their subtlety, yet predispose patients to an adverse outcome and subclinical ailing turns into evident failing after years.

Earlier studies have not differentiated patients with diabetes at the time of heart transplantation on the basis of their antidiabetic treatment $[4,5,12]$. In our study, diabetic patients treated with oral hypoglycaemic agents at the time of transplantation had the worst survival rate. Patients with diabetes, treated with diet alone, seem to show less severe metabolic disturbances and a shorter duration of disease than those treated with oral hypoglycaemic agents. Patients with diabetes treated by insulin at the time of heart transplantation showed similar survival rates compared to those treated with oral hypoglycaemic agents, it could be beneficial to optimize glycaemic control by early insulin substitution, if needed.

Both groups showed the same incidence of angiographically documented TxCAD. Furthermore, we did not observe any difference with regard to treatment of diabetes. The first finding is consistent with others reporting a similar incidence of TxCAD (32.8\% vs $32.0 \%$ ) after 4 years [5]. Compared to this study, the incidence of TxCAD in our study cohort was low and could have been influenced by our expanded use of cytolytic induction therapy with thymoglobuline [15]. As patients with diabetes in the general population are at increased risk of developing systemic atherosclerosis and coronary artery disease $[16,17,18]$, patients with diabetes and ischaemic CMP prior to transplantation could be more prone to develop TxCAD than others. In a study investigating late graft failure in renal transplant recipients, diabetes was a independent predictor of graft loss [14]. In contrast, another study failed to obtain a difference between renal transplant recipients with and without diabetes in terms of graft loss [19]. It could be likely, that either a follow-up period of 10 years is too short or our immunosuppressive protocol per se delays to a certain extent the development of atherosclerosis in coronary arteries [15, 20, 21, 22].

Patients with diabetes at the time of heart transplantation who received cytolytic induction therapy with thymoglobuline had a worse survival rate than patients without diabetes receiving cytolytic induction therapy with thymoglobuline. In general, cytolytic induction therapy with thymoglobuline has been shown to improve long-term survival in patients after heart transplantation mainly due to a reduction in incidence and severity of acute rejection as well as TxCAD [15]. However, it seems likely, that thymoglobuline alone might not reduce the impact of diabetes on long-term survival as the multifactorial process of diabetic microangiopathy and macroangiopathy relates to other mechanisms than induction of a more tolerant state. Patients with diabetes, who received cytolytic induction therapy with thymoglobuline showed the same low incidence of TxCAD as patients without diabetes with cytolytic induction therapy with thymoglobuline. Critics of induction therapy suggest that its use could be the most significant risk factor for CMV infection or disease, which we did not observe. The overall infection rate was comparable to others $[23,24]$. Patients without cytolytic induction therapy with thymoglobuline had a higher incidence of TxCAD, independent of diabetes at the time of heart transplantation.

The use of Tacrolimus is stated to relate to a higher incidence and a more severe form of diabetes after organ transplantation [25]. We did not observe any aggravation of diabetes in our study cohort. All patients with diabetes treated with Tacrolimus were alive without any signs of TxCAD after 4 years. As the number of patients with diabetes treated with Tacrolimus after heart transplantation is very small, one should be cautious to draw any definite conclusions from these findings.

Infectious complications were comparable in patients with and without diabetes. This is in contrast to renal transplants as well as to non-immunosuppressed patients with diabetes $[4,5,26,27]$. The higher state of immunosuppression after heart transplantation 
could overwhelm the impact of diabetes on infection. The severity and number of rejection episodes were also comparable, as reported in former studies $[4,5]$. Both groups did not differ in the kind and number of tumours. Different skin cancers were most common followed by lymphoma and lung cancer. Little is known of tumours in heart recipients with diabetes, however a large series of recipients without diabetes report similar data on malignancy $[15,28,29,30]$.

Limitations of the study. This analysis has all the drawbacks of a retrospective study. Results could have been influenced by the use of cytolytic induction therapy as compared to many other centres not using induction therapy. Additionally, the impact of Tacrolimus cannot be objectively evaluated, because of the low number and the shorter follow-up period in diabetic patients with Tacrolimus. It has to be mentioned that we focused on patients with diabetes at the time of heart transplantation. All patients, who developed post-transplant diabetes (to a certain extent related to immunosuppressive therapy) were not included in the group of patients with diabetes at the time of heart transplantation, since it was our primary intention to define risk stratification for preoperative diagnosis.

We conclude that diabetes at the time of heart transplantation adversely affects long-term survival rates in heart transplant recipients, but not the occurrence of TxCAD. The definite mechanisms of adverse survival in patients with diabetes are not clear. Facing the increasing number of patients with diabetes eligible for heart transplantation, we have to accept that this form of long-term palliation is associated with a less favourable outcome as compared with patients without diabetes. Nevertheless, from our data we believe that heart transplantation in patients with diabetes mellitus still is a valuable therapeutic option.

\section{References}

1. Griepp RB (1979) A decade of human heart transplantation. Transplant Proc 11:285-292

2. Lower RR, Szentpetery JQ, Thomas FT (1979) Selection of patients for cardiac transplantation. Transplant Proc 11:293-295

3. Badellino MM, Cavarocchi NC, Narins B et al. (1990) Cardiac transplantation in diabetic recipients. Transplant Proc 22:2384-2388

4. Ladowski JS, Kormos RL, Uretsky BF, Griffith BP, Armitage JM, Hardesty RL (1990) Heart transplantation in diabetic recipients. Transplantation 49:303-305

5. Munoz E, Lonquist JL, Radovancevic B et al. (1992) Longterm results in diabetic patients undergoing heart transplantation. J Heart Lung Transplant 11:943-949

6. Hamby RI, Zoneraich S, Sherman L (1974) Diabetic cardiomyopathy. JAMA 229:1749-1754

7. Garcia MG, McNarama PM, Gordon T, Kannel WB (1974) Morbidity and mortality in diabetic patients in the Framingham population: sixteen year follow-up study. Diabetes 23:105-111
8. Hopkins PN, Williams RR (1981) A survey of 246 suggested coronary risk factors. Atherosclerosis 40:1-52

9. Billingham ME, Cary NRB, Hammond ME et al. (1990) A working formulation for the standardization of nomenclature in the diagnosis of heart and lung rejection: Heart rejection study group. J Heart Transplant 9:587-593

10. Constanzo MR, Naftel DC, Pritzker MR et al. (1998) The Cardiac Transplant Research Database: Heart transplant coronary artery disease detected by coronary angiography: a multiinstitutional study of preoperative donor and recipient risk factors. J Heart Lung Transplant 17:744-753

11. Johnson JA, Kobashigawa JA (1995) Quantitative analysis of transplant coronary artery disease with use of intracoronary ultrasound. J Heart Lung Transplant 14:198-202

12. Tenderich G, Schulte-Eistrup S, Petzold R, Koerfer R (2000) Cardiac transplantation in patients with insulintreated diabetes mellitus. Exp Clin Endocrinol Diabetes 108:249-252

13. Jindal RM, Hjelmesaeth J (2000) Impact and management of posttransplant diabetes mellitus. Transplantation 70:SS58-SS63

14. Miles AMV, Sumrani NB, Horowitz R et al. (1998) Diabetes after renal transplantation. As deleterious as non-transplant-associated diabetes? Transplantation 65:380-384

15. Zuckermann A, Grimm M, Czerny M et al. (2000) Improved long-term results with thymoglobuline induction therapy after cardiac transplantation: a comparison of two different rabbit-antithymocyte globulines. Transplantation 69:1890-1898

16. Lee WL, Cheung AM, Cape D, Zinman B (2000) Impact of diabetes on coronary artery disease in women and men. Diabetes Care 23:962-968

17. Ganda OP (1985) Pathogenesis of macrovascular disease including the influence of lipids. In: Marble A, Krall LP, Bradley RF, Christlieb AR, Soeldner JS (eds) Joslin's diabetes mellitus. Lea and Febiger, Philadelphia, pp 217-250

18. Standl E, Schnell O (2000) A new look at the heart in diabetes mellitus: from ailing to failing. Diabetologia 43:1455-1469

19. Vesco L, Busson M, Bedrossian J et al. (1996) Diabetes mellitus after renal transplantation. Transplantation 61:1475-1478

20. Poston RS, Billingham M, Hoyt EG et al. (1999) Rapamycin reverses chronic graft vascular disease in a novel cardiac allograft model. Circulation 100:67-74

21. Valantine HA, Gao SZ, Menon SG et al. (1999) Impact of prophylactic immediate posttransplant ganciclovir on development of transplant atherosclerosis: a post hoc analysis of a randomized, placebo-controlled study. Circulation 100:61-66

22. Lamick R, Ballester M, Marti V et al. (1998) Efficacy of augmented immunosuppressive therapy for early vasculopathy in heart transplantation. J Am Coll Cardiol 32:413-419

23. Sarris GE, Moore KA, Schroeder JS et al. (1994) Cardiac transplantation: the Stanford experience in the cyclosporine era. J Thorac Cardiovasc Surg 108:240-251

24. Frist WH, Merill WH, Eastburn TE et al. (1990) Unique antilymphocyte serum versus OKT3 for induction immunotherapy after heart transplantation. J Heart Transplant 9:489-494

25. Montori V, Basu A, Erwin P, Velosa J, Gabriel SE, Kudva Y (2002) Posttransplantation diabetes: A systematic review of the literature. Diabetes Care 25:583-592

26. Shaffer O, Hammer SM, Monaco AP (1987) Infectious complications with the use of cyclosporine versus azathioprine after cadaver kidney transplantation. Am J Surg 153:381-386 
27. Scroggs MW, Wolfe JA, Bollinger R et al. (1987) Causes of death in renal transplant recipients. Arch Pathol Lab Med 111:983-987

28. Espana A, Redondo P, Fernandez AL et al. (1995) Skin cancer in heart transplant recipients. J Am Acad Dermatol $32: 458-465$
29. Penn I (1993) Tumors after renal and cardiac transplantation. Hematol Oncol Clin North Am 7:431-45

30. Rose AG, Viviers L, Odell JA (1992) Autopsy-determined causes of death following cardiac transplantation. A study of 81 patients and literature review. Arch Pathol Lab Med 116:1137-1141 